\title{
Public Health Workers' Knowledge, Attitude, and Practice Regarding COVID-19: The Impact of the Field Epidemiology Training Program in the Eastern Mediterranean Region
}

Sahar Sami ${ }^{1}$, MPH, MD; Faris Lami ${ }^{2}$, PhD; Hiba Abdulrahman Rashak ${ }^{3}$, MSc; Mohannad Al Nsour ${ }^{4}$, MD, MSc, PhD; Alaa Eid ${ }^{1}$, MD; Yousef Khader ${ }^{5}$, SCD; Salma Afifi $\mathrm{Sr}^{1}$, MD, MPH; Maisa Elfadul ${ }^{6}$, MD; Yasser Ghaleb ${ }^{7}, \mathrm{MSc}$; Hajer Letaief $^{8}$, MD; Nissaf Ben Alaya ${ }^{9}, \mathrm{PhD}$; Aamer Ikram ${ }^{10}, \mathrm{PhD}$; Hashaam Akhtar ${ }^{11}$, PhD; Abdelaziz Barkia Sr ${ }^{12}, \mathrm{MPH}$; Hana Ahmad Taha ${ }^{4}$, PhD; Reema Adam ${ }^{13}$, MAES; Khwaja Mir Islam Saeed $\mathrm{Sr}^{14}, \mathrm{PhD}$; Sami Almudarra ${ }^{15}, \mathrm{MD}, \mathrm{PhD}$; Mohamed Hassany ${ }^{1}$, MD; Hanaa Abu El Sood ${ }^{1}$, MD, MSc; Fazal ur Rahman ${ }^{16}$, FCPS; Falah Abdul-kader Saaed ${ }^{17}$, MD; Mohammed Sameer Hlaiwah ${ }^{17}$, MD

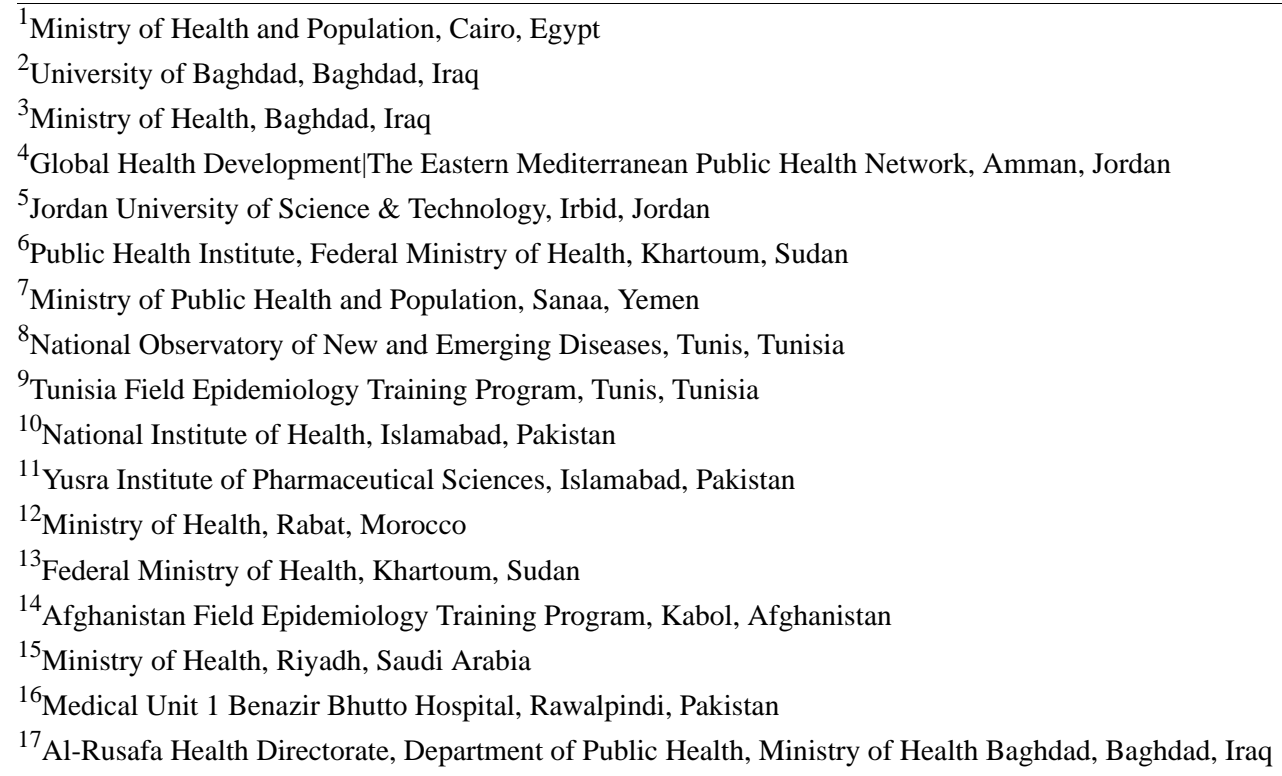

Corresponding Author:

Sahar Sami, MPH, MD

Ministry of Health and Population

3a Magles Elshaab street

Cairo

Egypt

Phone: 2001001541035

Email: saharmohp@yahoo.com

\section{Abstract}

Background: Globally, there is a growing need for public health professionals skilled in preventing and responding to the surge of emerging and re-emerging infectious diseases. This is particularly important to the Eastern Mediterranean countries that are facing emergencies in addition to the increased public health risks of unprecedented scale during the COVID-19 pandemic. Public health professionals are instrumental in responding to the COVID-19 pandemic in terms of detecting and monitoring new cases, conducting investigations, tracing contacts, ensuring patients are being tested, applying isolation and quarantine protocols, providing up-to-date information, educating the community, and producing statistics and models to track disease progression.

Objective: This study aims to compare knowledge, attitude, and practice (KAP) regarding COVID-19 between public health workers (PHWs) that attended the Field Epidemiology Training Program (FETP trained) and those who did not attend FETP (non-FETP trained). 
Methods: A multicountry cross-sectional survey was conducted among PHWs who participated in the COVID-19 pandemic in 10 countries in the Eastern Mediterranean Region. An online questionnaire that included demographic information and KAP regarding the COVID-19 pandemic was distributed among PHWs. The scoring system was used to quantify the answers; bivariate and multivariate analyses were performed to compare FETP-trained with non-FETP-trained PHWs.

Results: Overall, 1337 PHWs participated, with $835(62.4 \%)<40$ years of age and $851(63.6 \%)$ male participants. Of them, $423(31.6 \%)$ were FETP trained, including $189(44.7 \%)$ at an advanced level, $155(36.6 \%)$ at an intermediate level, and 79 (18.7\%) had basic level training. Compared to non-FETP-trained participants, FETP-trained participants were older and had higher KAP scores. FETP participation was low in infection control and public health laboratories. KAP mean scores for intermediate-level attendees were comparable to the advanced level.

Conclusions: FETP-trained participants had better KAP than non-FETP-trained PHWs. Expanding the intermediate level, maintaining the rapid response training, and introducing the laboratory component are recommended to maximize the benefit from the FETP. Infection control, antimicrobial resistance, and coordination are areas where training should be included.

(iproc 2022;8(1):e36491) doi: $10.2196 / 36491$

\section{KEYWORDS}

knowledge; attitude; practice; COVID-19; Field Epidemiology Training Program

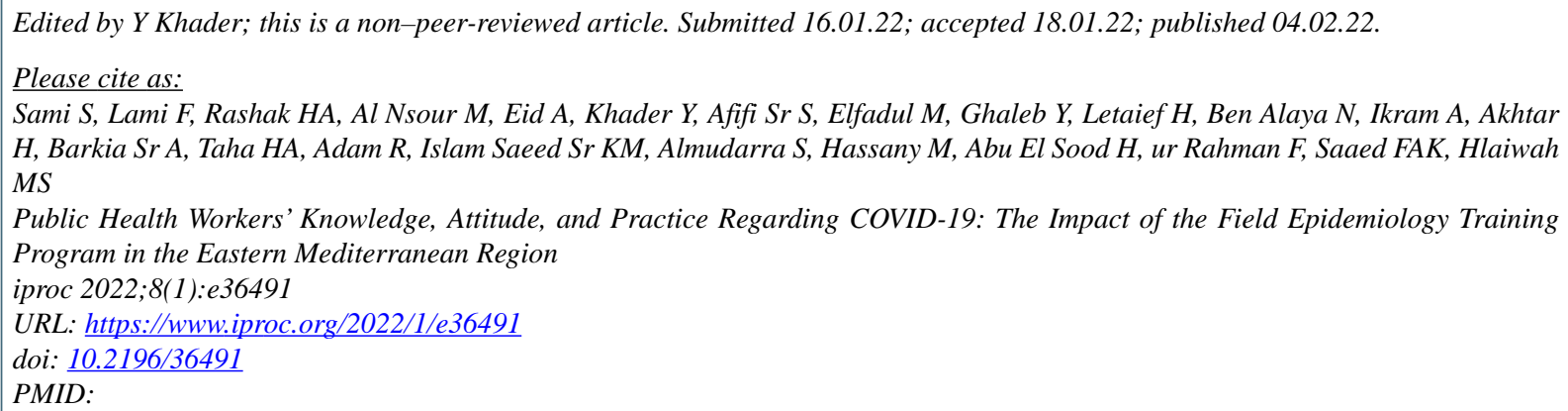

(C) Sahar Sami, Faris Lami, Hiba Abdulrahman Rashak, Mohannad Al Nsour, Alaa Eid, Yousef Khader, Salma Afifi Sr, Maisa Elfadul, Yasser Ghaleb, Hajer Letaief, Nissaf Ben Alaya, Aamer Ikram, Hashaam Akhtar, Abdelaziz Barkia Sr, Hana Ahmad Taha, Reema Adam, Khwaja Mir Islam Saeed Sr, Sami Almudarra, Mohamed Hassany, Hanaa Abu El Sood, Fazal ur Rahman, Falah Abdul-kader Saaed, Mohammed Sameer Hlaiwah. Originally published in Iproceedings (https://www.iproc.org), 04.02.2022. This is an open-access article distributed under the terms of the Creative Commons Attribution License (https://creativecommons.org/licenses/by/4.0/), which permits unrestricted use, distribution, and reproduction in any medium, provided the original work, first published in Iproceedings, is properly cited. The complete bibliographic information, a link to the original publication on https://www.iproc.org/, as well as this copyright and license information must be included. 